\title{
Energy Efficiency Maximization for Wireless Sensor Networks *
}

\author{
Inwhee Joe \\ College of Information and Communications \\ Hanyang University \\ Seoul, Korea \\ iwjoe@hanyang . ac.kr
}

\begin{abstract}
Because of the remote nature and the size of sensor nodes, they rely on limited battery energy that cannot be replenished in many applications. Thus, low power consumption technology is a major issue in wireless sensor networks in order to prolong system lifetime. In this paper, we propose to maximize energy efficiency in wireless sensor networks using optimal packet length in terms of power management and channel coding. The use of power management cannot improve energy efficiency, but it saves a lot of energy because the transceiver is turned off while it is not used. Also, we evaluate optimal packet length without power management in such that the energy efficiency can be maximized. Finally, we show that the BCH code for channel coding can improve energy efficiency significantly compared to the convolutional code.
\end{abstract}

\section{Introduction}

A wireless sensor network has been recognized as an important technology in realizing ubiqutous computing. In an early stage, it is designed for an unmanned surveillance system, monitoring military tendency in terrain where access is difficult. Recently, a sensor network is used for remote sensing in a number of areas including intelligence traffic system, factory process control or environment control of intelligent building. The concept of sensing and wireless connection promises many new application areas. Sensor networks provide a new kind of capability that enables us to observe and interact with physical phenomenon in real time and in detail that was unobtainable before.

In the wireless sensor network, a large number of sensor nodes are deployed either inside the phenomenon or very close to it. Each node consists of sensing, data processing, and communication components. Sensor nodes are embedded with an onboard processor. When events of interest are detected by sensor nodes, they use their processing power to carry out simple computations and transmit only the required data to a remote base station instead of sending the raw data.

\footnotetext{
* This work was supported in part by grant No. IITA-2005-C1090-0501-0022 from the ITRC Support Program of the Ministry of Information and Communication, and in part by grant No. HY-2003-T from the Research Fund of Hanyang University.
}

\footnotetext{
Please use the following format when citing this chapter:
} 
The wireless sensor network is quite different from the traditional wireless networks [6]. It has a large number of sensor nodes and they are densely deployed. The distance between neighbor nodes is shorter compared to other wireless networks. The data rate and mobility in the wireless sensor network are low. Because of the remote nature and the size of the individual nodes, they rely on limited battery energy that cannot be replenished for most wireless sensor networks. In many cases, sensor nodes are placed in the field for years at a time without maintenance or human intervention of any kind. Thus, low power consumption technology is a major issue in wireless sensor networks in order to prolong system lifetime.

In this paper, we show that energy efficiency can be improved by optimal packet length at the data link layer. The optimal packet length is obtained in terms of energy efficiency. Also, we present that the use of power management for improving energy efficiency is not justified. Then, we show optimal packet length without power management. Power management is to turn off the transceiver when it is in the idle state. Finally, we evaluate that the $\mathrm{BCH}$ code for error control can improve energy efficiency significantly compared to the convolutional code.

The structure of this paper is as follow. In Section 2, we compute the probability of error as a function of neighbor distance between sensor nodes. In Section 3 , we compare the optimal packet length with power management versus without it in terms of energy efficiency. In Section 4, we show that the BCH code can improve energy efficiency significantly compared to the convolutional code. Finally, we conclude this paper in Section 5.

\section{Channel Estimation}

In this section, we estimate the raw channel BER (Bit Error Rate) for typical wireless sensor networks. The probability of bit error $\left(P_{b}\right)$ under a FSK modulated Rayleigh fading channel model for wireless sensor networks is defined as follows [7]:

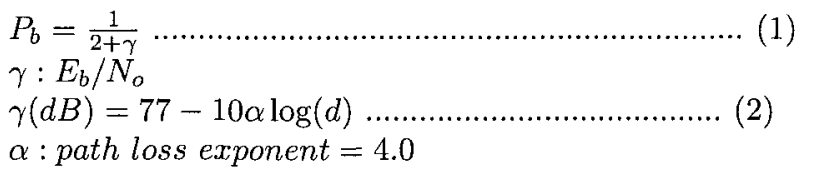

where $\gamma$ is the average received bit energy to noise ratio. According to the receiver implementation, $\gamma$ depends on the neighbor distance $d$ between sensor nodes. The path loss is the average propagation loss as a function of the distance $d$ on the order of $\alpha$.

We assume the RFM-TR1000 transceiver with $-9 \mathrm{dBm}$ output power, $7.5 \mathrm{~dB}$ noise figure and $6 \mathrm{~dB}$ implementation losses. Fig. 1 presents the probability of bit error by Equation (1) according to the neighbor distance with path loss exponent $\alpha=4$ in the worst case design. Normally, most sensor nodes are separated 


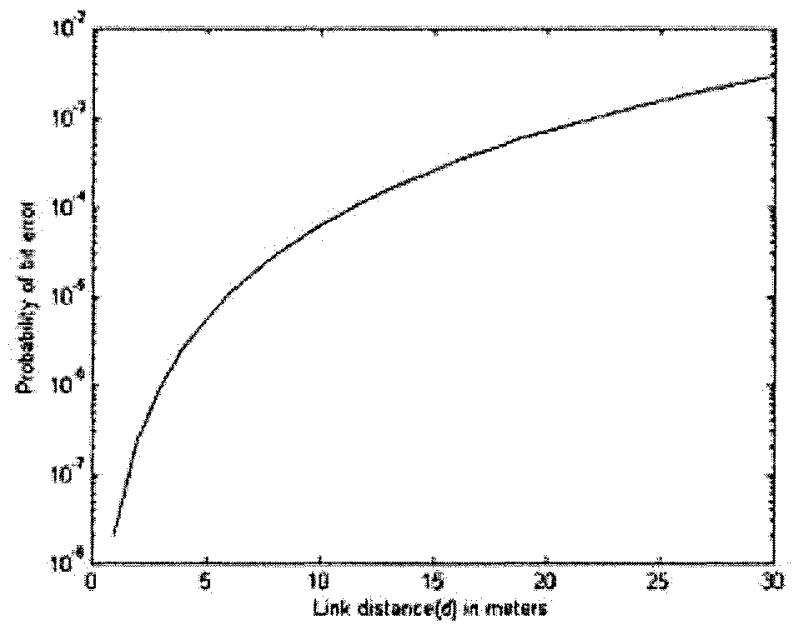

Fig. 1. Probability of bit error as a function of neighbor distance

from each other at a distance between 10 and $20 \mathrm{~m}$ in wireless sensor networks. Therefore, the estimated channel BER lies in the range of $2 \times 10^{-4}$ and $3 \times 10^{-3}$ in this case.

\section{Power Management and Optimal Packet Length}

Due to the time-varying nature of the wireless channel, the throughput is very sensitive to the packet length. It is well known that the variable packet length with channel conditions can result in significant throughput improvement [4]. For example, if the wireless channel condition becomes worse, the smaller packet length is more desirable because the error rate is higher and the larger packets are likely to fail in transit. However, the variable packet length is not appropriate for wireless sensor networks due to the complexity. Even if the throughput is lower, we support the use of the fixed packet length for energy efficiency in wireless sensor networks, which is more important in this case. Moreover, we propose to use the optimal fixed packet length in terms of maximizing the energy efficiency.

Power management is to turn off the transceiver to reduce energy consumption when it is in the idle state. With the use of power management, it is possible to accomplish significant energy savings. However, since sensor nodes normally communicate using short packets, energy efficiency could be reduced instead due to the dominance of start-up energy. The energy efficiency equation is defined by [1]

$\eta=E_{t h} \cdot R$

$\eta$ : energy efficiency 
$E_{\text {th }}$ : energy throughput

$R$ : reliability

where the energy throughput $E_{t h}$ represents the ratio of energy consumed for actual data transmission to entire packet transmission, and the reliability $R$ represents the successful packet reception rate. That is, the energy efficiency $\eta$ can be obtained from the energy throughput multiplied by the reliability, which means how much energy is actually used for successful data transmission.

According to the use of power management, Equation (3) can be expressed in more concrete form. While Equation (4) represents energy efficiency as a function of packet length with power management, Equation (5) represents energy efficiency without power management as follows:

$\eta=\frac{E_{c} l}{E_{c}(l+h)+E_{s}} \cdot(1-P E R)$

$\eta=\frac{l}{l+h} \cdot(1-P E R)$

$E_{c}:$ communication energy consumption

$E_{s}:$ start - up energy consumption

$l:$ payload length

$h$ : header length

$P E R$ : packet error rate

We compare energy efficiency with power management versus without it in Figs. 2 and 3. Fig. 2 shows energy efficiency for the neighbor distance $10 \mathrm{~m}$, while Fig. 3 shows energy efficiency for the neighbor distance $20 \mathrm{~m}$. From these results, we can find out that the use of power management cannot improve energy efficiency. However, if power management is not used, it causes a lot of energy waste. In summary, even if power management cannot improve energy efficiency, it should be employed in order to minize the waste of energy.

It is apparent that if the packet length is too small, it suffers from an efficiency problem due to the larger overhead. On the other hand, if the packet length is too large, it experiences higher packet error rates especially for the wireless channel with high error rates. Since an error packet means a total loss of energy consumed for the packet transmission, it also suffers from an efficiency problem in this case. Therefore, there exists an optimal packet length in the sense of maximizing the energy efficiency. In particular, when energy efficiency is at peak, the optimal payload length is 280 bits for $10 \mathrm{~m}$ of neighbor distance and it is 60 bits for $20 \mathrm{~m}$ of neighbor distance, if power management is applied. Also, the maximum values of energy efficiency are measured by 0.88 and 0.62 , respectively.

\section{Channel Coding and Optimal Packet Length}

Channel coding is one of typical approaches to increase link reliability in the design of wireless networks with poor channel conditions. Before it is applied to wireless sensor networks, we should check first if channel coding can improve 


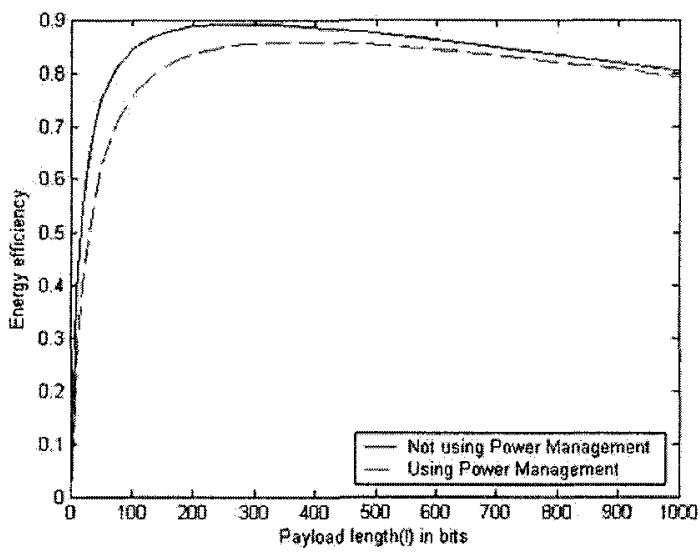

Fig. 2. Energy efficiency as a function of payload length for neighbor distance $d=10 \mathrm{~m}$ and $h=16$ bits

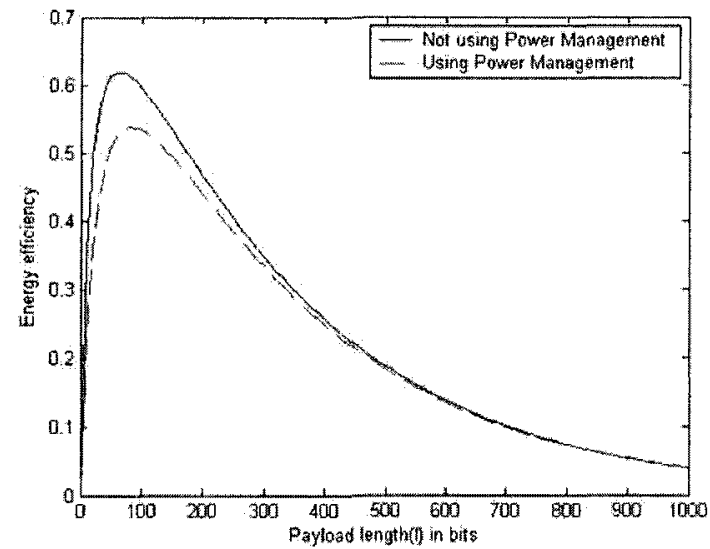

Fig. 3. Energy efficiency as a function of payload length for neighbor distance $d=20 \mathrm{~m}$ and $h=16 b i t s$ 
energy efficiency. In this section, energy efficiency is compared between the BCH code and convolutional code. Turbo and LDPC codes are not considered here, because their iterative decoding process consumes a lot of energy. It turns out that they are not appropriate for wireless sensor networks due to the very limited energy.

The energy efficiency of the rate- $1 / 2$ convolutional code is given by

$\eta=\frac{E_{c}\left(\frac{n}{2}-h\right)}{E_{c} n+E_{s}+E_{d e c}} \cdot\left(1-\dot{P}_{b}\right)^{n}$

$\dot{P}_{b}$ : Probability of bit error for convolutional code

where $n$ is the packet length, $h$ is the header length, and $E_{d e c}$ is the decoding energy consumed at the receiver. The upper bound on the probability of bit error for the convolutional code can be expressed by

$\dot{P}_{b}<\frac{1}{k} \sum_{d=d f r e e}^{\infty} \beta d P(d)$

where $d$ denotes the Hamming distance in the trellis diagram between some path and the all-zero path, $d_{\text {free }}$ is the minimum free distance, $\beta_{d}$ is the cofficient of the first derivative of the transfer function $T(N, D)$ with respect to $\mathrm{N}$, and $P(d)$ is the first-event error probability.

For the $\mathrm{BCH}$ code, we first consider a binary $\mathrm{BCH}$ code with hard decision. The encoder adds $\tau$ parity bits to the $l$ payload and $h$ header bits. As a result, the packet length $n$ equals to $l+h+\tau$. The energy efficiency of the $\mathrm{BCH}$ code with the $t$ error correction capability is given by

$\eta=\frac{E_{c}(n-h-\tau)}{E_{\mathrm{c}} n+E_{s}+E_{d e c}} \cdot \sum_{j=0}^{t}\left(\begin{array}{c}n \\ j\end{array}\right) P_{b}^{j}\left(1-P_{b}\right)^{n-j}$

Equation (9) gives the decoding energy for a $t$ error correction binary BCH code of length $n$, defined as

$E_{d e c}=\left(2 n t+2 t^{2}\right)\left(E_{a d d}+E_{\mu l t}\right)$

$E_{a d d}=3.3 * 10^{-5} \mathrm{~m}(\mathrm{~mW} / \mathrm{MHz})$

$E_{m u l t}=3.7 * 10^{-5} \mathrm{~m}^{3}(\mathrm{~mW} / \mathrm{MHz})$

$\tau=m t(\tau \leq m t)$

$\tau:$ number of parity bits

$t$ : error correction capability

where $E_{a d d}$ and $E_{m u l t}$ are the energy consumptions in the addition and multiplication under $\operatorname{GF}\left(2^{m}\right)\left(m=\left|\log _{2} n+1\right|\right)$.

Fig. 4 shows energy efficiency for the convolutional code using Equation (6) versus the $\mathrm{BCH}$ code using Equation (8) each at the channel BER $10^{-3}$. From these results, we observe that the use of channel coding can improve energy efficiency significantly. It is also shown that the energy efficiency of the convolu- 


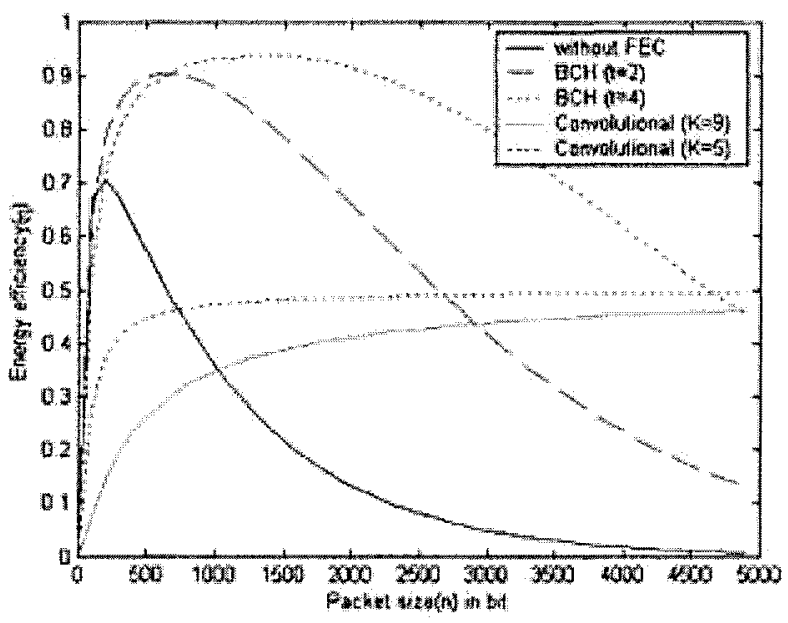

Fig. 4. Energy efficiency as a function of packet length for comparison of BCH versus Convolutional codes with $h=16$ bits

tional code is only half or so, compared to that of $\mathrm{BCH}$ code. The reason for the lower energy efficiency is that the convolutional code causes a larger overhead in that for each packet the half of it is data and the rest is redundancy. In other words, since more than half of the total energy is consumed for redundancy and control overhead, it leads to a substantial degradation in terms of energy efficiency. On the other hand, the $\mathrm{BCH}$ code introduces much less redundancy unlike the convolutional code.

Since high energy efficiency is required for wireless sensor networks, the use of the $\mathrm{BCH}$ code is a viable solution. As compared to the case without the use of channel coding, it would rather improve the energy efficiency. Also, we find out that the energy efficiency is reduced in case of the BCH code as the error correction capability $t$ is increased. In particular, the optimal packet length is 700 bits and its energy efficiency is 0.90 for the error correction capability of 4 , while the optimal packet length is 1400 bits and its energy efficiency is 0.93 for the error correction capability of 2 . With the use of the $\mathrm{BCH}$ code, the energy efficiency can be increased up to 0.93 from 0.7 , which is the maximum value of the case without the use of channel coding.

\section{Conclusions}

Because of the remote nature and the size of sensor nodes, they rely on limited battery energy that cannot be replenished in many applications. Thus, the energy efficiency is a critical factor in wireless sensor networks in order to prolong system lifetime. 
Due to the time-varying nature of the wireless channel, the throughput is very sensitive to the packet length. It is well known that the variable packet length with channel conditions can result in significant throughput improvement. However, the variable packet length is not appropriate for wireless sensor networks due to the complexity. Even if the throughput is lower, we support the use of the fixed packet length for energy efficiency in wireless sensor networks, which is a more important factor in this case. Moreover, we propose to use the optimal fixed packet length in terms of maximizing the energy efficiency.

In this paper, we have shown that energy efficiency can be maximized by optimal packet length in wireless sensor networks. The use of power management cannot improve energy efficiency, but it saves a lot of energy because the transceiver is turned off while it is not used. Also, we have found out that the $\mathrm{BCH}$ code can improve energy efficiency significantly compared to the convolutional code. From our test results, we have concluded that energy efficiency can be improved substantially through optimal packet length and channel coding.

\section{References}

1. Y. Sankarasubramaniam, I.F. Akyildiz and S.W. McLaughlin, "Packet Size Optimization and its Implications on Error Control for Sensor Networks," Proceedings of the 1st IEEE International Workshop on Sensor Network Protocols and Applications, May 2003.

2. E. Shih, B.H. Calhoun, S. Cho, and A.P. Chandrakasan, "Energy-Efficient Link Layer for Wireless Microsensor Networks," IEEE Computer Society Workshop on VLSI 2001, pp. 16-21, April 2001.

3. K. Sohrabi, J. Gao, V. Ailawadhi, and G.J. Pottie, "Protocols for Self-Organization of a Wireless Sensor Network," IEEE Personal Communications Magazine, pp. 1627 , October 2000.

4. I.F. Akyildiz and I. Joe, "A New ARQ Protocol for Wireless ATM Networks," Proceedings of IEEE International Conference on Communications ICC'98, pp. 11091113, June 1998.

5. G.J. Pottie and W.J. Kaiser, "Wireless Integrated Network Sensors," Communications of ACM, Vol. 43, No. 5, pp. 51-58, May 2000.

6. I.F. Akyildiz, W. Su, Y. Sankarasubramaniam, and E. Cayirci, "A Survey on Sensor Networks," IEEE Communications Magazine, pp. 102-114, August 2002.

7. T. Rappaport, "Wireless Communications: Principles and Practice," Prentice Hall, 2002.

8. I. Joe, "A Novel Adaptive Hybrid ARQ Scheme for Wireless ATM Networks," ACMBaltzer Wireless Networks Journal, Vol. 6, No. 3, pp. 211-219, June 2000. 